\title{
Regenerative ability of augmented bone in rat calvarial guided bone augmentation model
}

Katsuyoshi Tsunori1)2) / Naoya Tsukune ${ }^{3)}$ / Tatsuya Kubota ${ }^{3)}$ ( Akira Hasuike ${ }^{122}$ / Shuichi Sato ${ }^{12)}$ 1 Department of Periodontology, Nihon University School of Dentistry, Tokyo, Japan

2 Division of Advanced Dental Treatment, Dental Research Center, Nihon University School of Dentistry, Tokyo, Japan

3 Division of Applied Oral Sciences, Nihon University Graduate School of Dentistry, Tokyo, Japan

\section{Abstract}

Purpose: Guided bone regeneration (GBR) is the most widely used technique to regenerate and augment bones. Even though augmented bones (ABs) have been examined histologically in many studies, few studies have been conducted to examine the biological potential of these bones and the healing dynamics following their use. Moreover whether the bone obtained from the GBR procedure possesses the same functions as the existing autogenous bone is uncertain. In particular, little attention has been paid to the regenerative ability of GBR bone. Therefore, the present study histologically evaluated the regenerative capacity of $A B$ in the occlusive space of a rat guided bone augmentation (GBA) model.

Methods: The calvaria of 30 rats were exposed, and plastic caps were placed on the right of the calvaria in 10 of the 30 rats. After a 12 week healing phase, critical-sized calvarial bone defects (diameter: $5.0 \mathrm{~mm}$ ) were trephined into the dorsal parietal bone on the left of the calvaria. Bone particles were harvested from the $A B$ or the cortical bone (CB) using a bone scraper and transplanted into the critical defects.

Results: The newly generated bone at the defects' edge was evaluated using micro-computed tomography (micro-CT) and histological sections. In the micro-CT analysis, the radiopacity in both the augmented and the CB groups remained high throughout the observational period.

In the histological analysis, the closure rate of the CB was significantly higher than in the AB group. The numbers of cells positive for runtrelated transcription factor 2 (Runx2) and tartrate- resistant acid phosphatase (TRAP) in the $A B$ group were larger than in the $C B$ group

Conclusions: The regenerative capacity of $A B$ in the occlusive space of the rat GBA model was confirmed. Within the limitations of this study, the regenerative ability of the $A B$ particulate transplant was inferior to that of the $\mathrm{CB}$ particulate transplant.

\section{Background and Aim}

Guided bone regeneration (GBR) is the most widely used technique to regenerate and augment bones. Even though augmented bones have been examined histologically in many studies, few studies have been conducted to examine the biological potential of these bones and the healing dynamics following their use. Moreover, whether the bone obtained from the GBR procedure possesses the same functions as the existing autogenous bone is uncertain. In particular, little attention has been paid to the regenerative ability of GBR bone.

The present study histologically evaluated the regenerative capacity of augmented bone in the occlusive space of a rat guided bone augmentation (GBA) model.

\section{Methods and Materials}

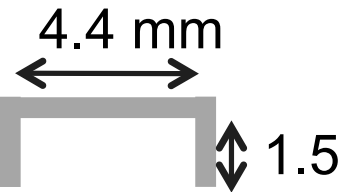
capacity $22.8 \mathrm{~mm}^{3}$

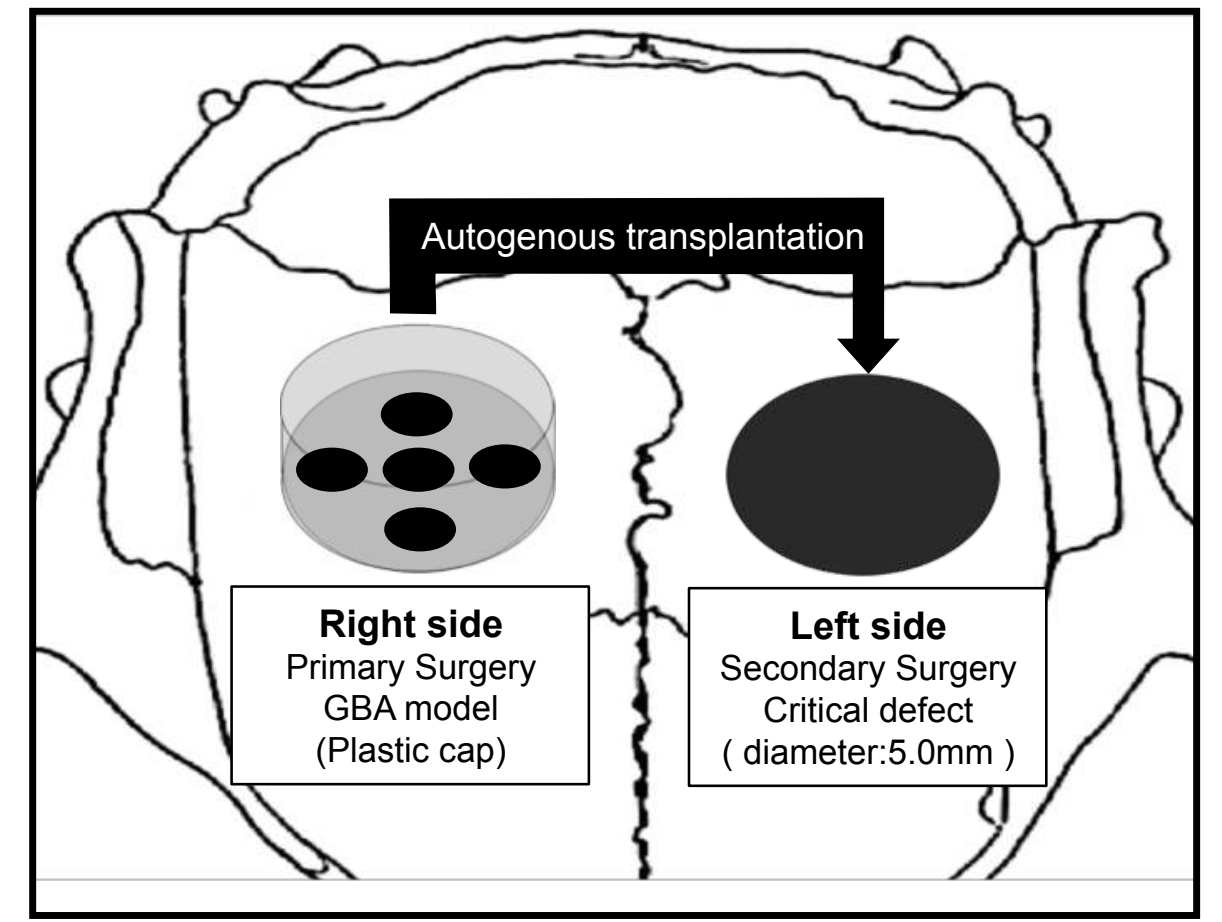

Thirty 8-week-old male Fischer rats were used. In an initial operation, a standardized plastic cap was put in place, and bone augmentation was performed. In a second operation 12 weeks later, a 5-mm-diameter bone defect was formed in the right parietal bone as the recipient site. The plastic cap was removed. In the experimental group, the recipient site was filled with newly augmented bone tissue as autotransplant bone ( $A B$ group). The recipient site was not filled in the control group. A sham operation was performed using autogenous bone from the left parietal bone to fill the bone defect (CB group). Repeated micro-computed tomography (CT) imaging was performed from 1 to 12 weeks after the second surgery. The defect sites were removed along with the surrounding bone and soft tissues, stained with hematoxylin - eosin and immnunostaining.

\section{Results}
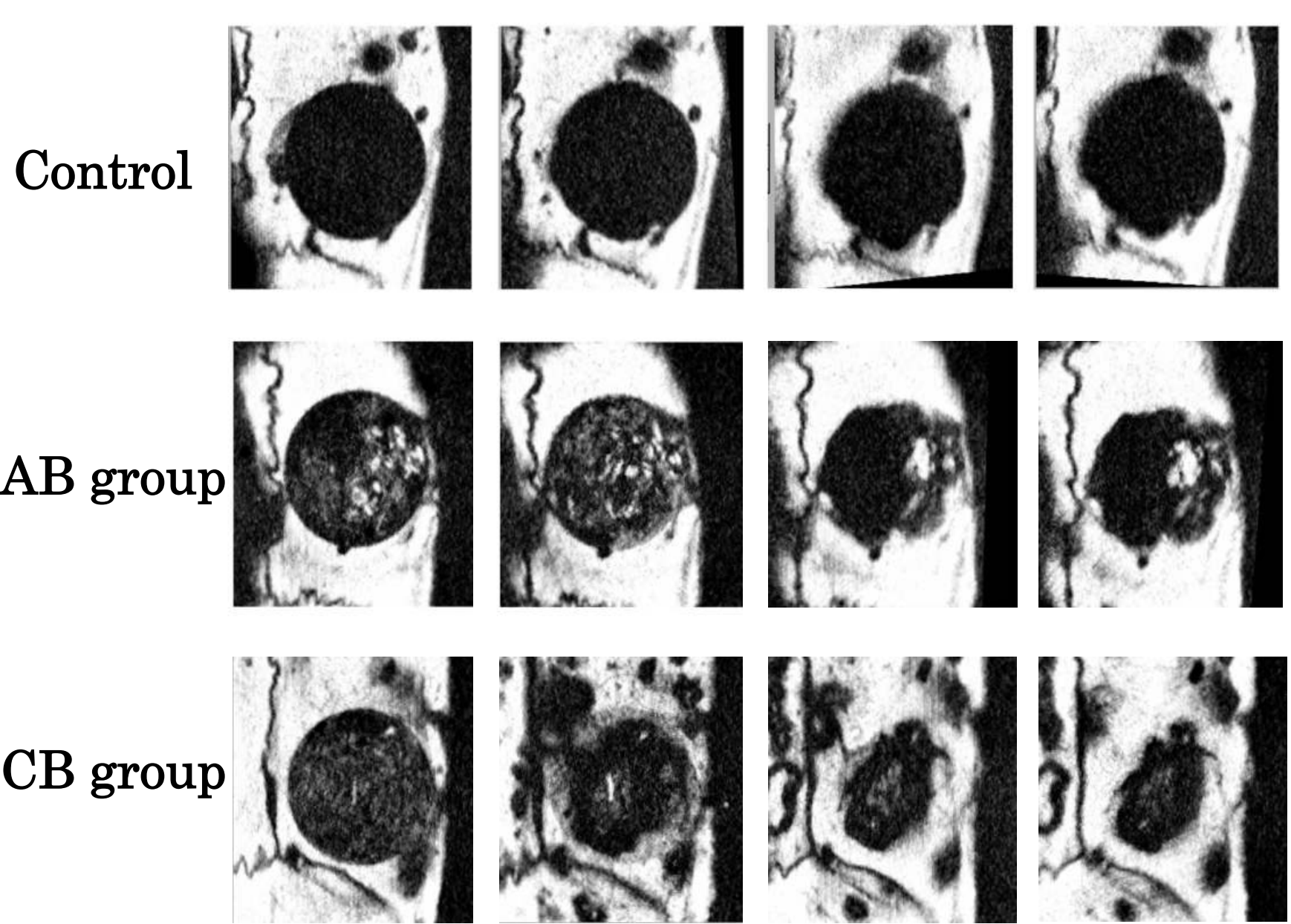

0 weeks

4 weeks

8 weeks

12 weeks

Micro-CT image of critical defects in the $A B$ group $C B$ group and control group at $0,4,8$ and 12 weeks after secondary surgery.

$\mathrm{BV}\left(\mathrm{mm}^{3}\right)$

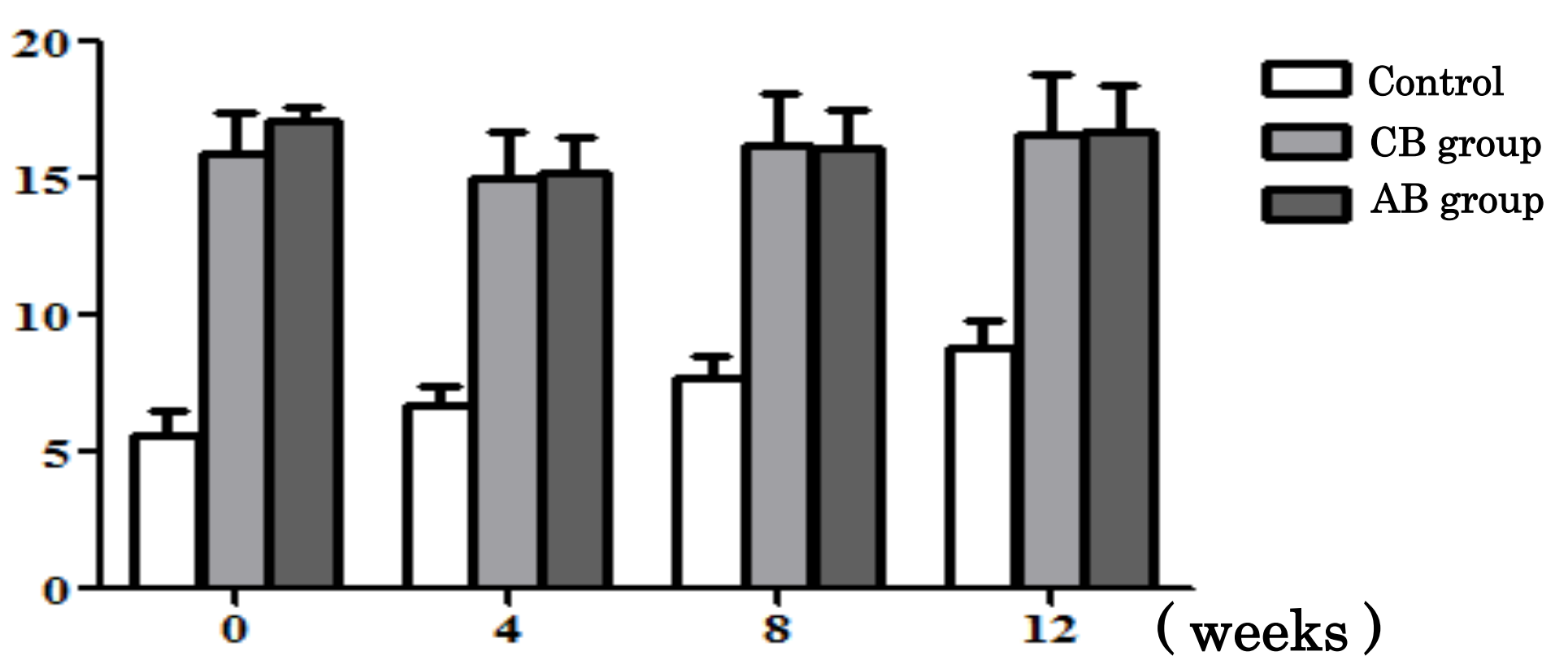

Statistical summary ( mean \pm SD ) for BV in critical defects obtained from Micro-CT.

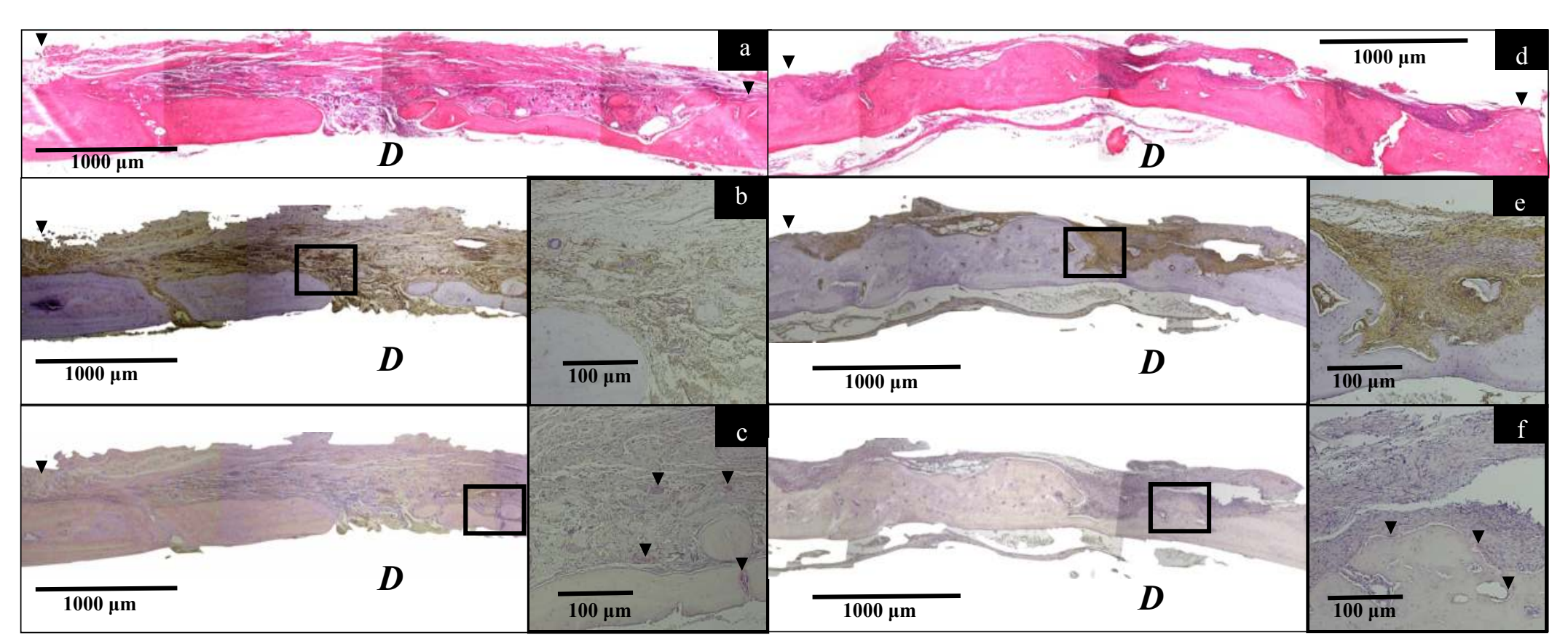

Representative histological observation of the critical defects at 12 weeks in the $C B$ group. Representative histological observation of the critical defects at 2 weeks in the $C B$ group.
a) $A B$ group; $-\mathrm{H}$ staining, b) $A B$ group; immunohistochemistry for Runx2, c) AB group; TRAP staining
d) $C B$ group; $H-E$ staining, e) $C B$ group; immunohistochemistry for Runx2, f) CB group; TRAP staining $(\downarrow$ :positive reaction, $D:$ dura, $\mathbf{\nabla}$ :edge of bone defect).

The newly generated bone at the defects' edge was evaluated using micro-computed tomography (micro-CT) and histological sections. In the micro-CT analysis, the radiopacity in both the augmented and the $\mathrm{CB}$ groups remained high throughout the observational period. In the histological analysis, the closure rate of the cortical bone was significantly higher than in the $A B$ group. The numbers of cells positive for runt-related transcription factor 2 and tartrate-resistant acid phosphatase in the $A B$ group were larger than in the CB group.

\section{Conclusions}

In conclusion, the regenerative capacity of augmented bone in the occlusive space of a rat GBA model was confirmed. Within the limitations of this study, the regenerative ability of the augmented bone particulate transplant was found to be inferior to that of a cortical bone particulate transplant.

\section{References}

Yutaka Yamada, Shuichi Sato, Hiroaki Yagi, Hisashi Ujiie, Sanae Ezawa, Koichi Ito (2012) Correlation in the densities of augmented and existing bone in guided bone augmentation. Clin Oral Implants Res 23, 837-845. 